\title{
Temporal evolution of CFC 11 and CFC 12 concentrations in the ocean interior
}

\author{
Peter Beining \\ Institut für Meereskunde an der Universität Kiel, Kiel, Germany \\ Wolfgang Roether \\ Institut für Umweltphysik der Universität Bremen, Bremen, Germany
}

\begin{abstract}
We study the temporal evolution of concentrations of the chlorofluorocarbons CFC 11 and CFC 12 in the ocean, under the assumption of circulation and mixing being invariant in time. This allows us to define a time-invariant age distribution for a given point in the ocean, where the age is defined as time since the last contact with the atmosphere occurred. This concept is evaluated for a number of fundamental situations. We deduce a tendency for low CFC 11 and CFC 12 concentrations in advective regimes to increase exponentially in time and for concentrations near to a solubility equilibrium with atmospheric concentrations to increase rather more linearly. The apparent saturations, i.e., the ratios of interior to mixed-layer CFC concentrations, increase monotonically in time, typical rates being 5-10\% per decade. The theoretical results are compatible with time trends found in repeated CFC observations in the ocean. Diagrams on the temporal evolution for different age distributions are presented for the period 1970-2000, which can serve as a general orientation. The diagrams furthermore can provide time corrections for quasi-synoptic evaluation of CFC observations taken over an extended period of time and assist in constructing time-dependent CFC boundary conditions for numerical models of ocean circulation.
\end{abstract}

\section{Introduction}

Transient tracers are of interest in oceanography because their oceanic distributions can provide information complementary to that obtained from classical hydrographic data, relevant topics being timescales and patterns of thermohaline circulation and diapycnal or isopycnal mixing. Two such tracers are the chlorofluorocarbons (CFCs) CFC 11 and CFC 12 [Bullister, 1989]. Their release into the environment started in the early 1940s, and atmospheric CFC concentrations have been increasing since. Concentrations in the troposphere are rather uniform spatially, except for a certain drop from the northern into the southern hemisphere. Atmosphere-ocean gas exchange brings about near-solubility equilibrium concentrations in the ocean mixed layer. Subsequently, circulation and mixing transfer the two compounds into deeper layers. Conservative behavior in the ocean is generally assumed. Advantageous features of the two tracers are that the time-dependent ocean surface concentrations are fairly well known, that concentrations down to a few per mil of current mixed-layer levels can be detected, and that, at not too low concentrations, measurement precision is high, i.e., of the order of $1 \%$ [Bullister and Weiss, 1988].

CFC 11 and CFC 12 are useful in water mass analysis, enabling in particular a distinction between old water (low concentration) and young water (higher concentration). A speciality is that until about 1980 , the CFC 11 to CFC 12 ratios in the atmosphere have continuously been rising, providing a calendar-year tag for the waters descending from the mixed

Copyright 1996 by the American Geophysical Union.

Paper number 96JC00987.

0148-0227/96/96JC-00987\$09.00 layer [Weiss et al., 1985; Wallace et al., 1994; Doney and Bullister, 1992]. Later on, the ratios decreased slowly, which introduced ambiguity in the tagging. A further limitation is that interior-ocean mixing (unless CFC-free water is admixed) commonly blurs the age signal rather heavily [Pickart et al., 1989; Smethie, 1993], the resulting CFC ratios being biased toward components with a higher CFC concentration, i.e., young ones. Mostly, therefore, the CFC-ratio "age" is of a parametric nature. A further use of CFC data is to extract information by comparing observed distributions with ones simulated by ocean circulation models. For a correct simulation the model must reproduce the Lagrangian penetration of waters from the ocean surface into the interior over the period of the CFC transient, i.e., the past few decades. This allows specific checks on model performance, in particular with respect to thermohaline circulation [Roether et al., 1994].

A problem is that CFC observations in the ocean are, and will always be, scarce in space and time. Therefore water mass analysis typically has to work with nonsynoptic data, which cause problems in view of the transient distribution of the CFCs. Similar problems arise in evaluations by means of oceanic circulation models, whenever time histories of CFC concentrations at inner-ocean model boundaries are to be prescribed. To construct these, observations at one point in time only are available as a rule. A solution has been sought by using the assumption that "apparent saturations," i.e., the ratios of interior-ocean CFC concentrations to the concurrent ones in the mixed layer, are sufficiently invariant in time [Gordon et al., 1992].

With the aim of providing orientation for these problems we assess in the present paper the temporal evolution of concentrations of the two CFCs in the ocean in quite general terms. 
We start by developing the concept of an age distribution for a stationary ocean. Subsequently, we select age distributions believed to be characteristic of, or limiting cases for, the actual ocean. For these, time curves of CFC concentrations, apparent saturations, and CFC 11 to $\mathrm{CFC} 12$ ratios are presented. The results are compared to some available repeated observations of CFC concentrations. Finally, some applications of our results are considered. We specifically deal with the southern hemisphere ocean, but the results are sufficiently general to be applicable also to the northern hemisphere.

\section{Theoretical Evolution of CFC Concentrations in a Stationary Ocean}

Consider a water parcel at a certain point in space and time in the ocean interior. It will be a mixture of components that each passed through the mixed layer at a specific source location and at a specific time prior to the actual point in time; the time difference since last contact with the atmosphere is denoted component age. If as a first approximation we assume that circulation and mixing are invariant in time, the same will hold for the composition of the parcel, i.e., the source locations and ages of the components. If we furthermore assume mixedlayer CFC concentrations $\left(c_{m l}{ }_{l}\right)$ to be in a solubility equilibrium with the atmosphere, the composition will uniquely determine the CFC concentration in the water parcel at any time. We write the relationship as

$$
c\left(t_{\mathrm{obs}}\right)=\int_{0}^{\infty} \frac{d g(t)}{d t} c_{m . l .}\left(t_{\mathrm{obs}}-t\right) d t=\int_{0}^{\infty} c_{m . l}\left(t_{\mathrm{obs}}-t\right) d g
$$

where $t_{\text {obs }}$ is the actual time of observation and $d g(t) / d t$ is the age distribution $([d g(t) / d t] d t$ is the fraction with age between $t$ and $t+d t$, with norm $\left.\int_{0}^{\infty}[d g(t) / d t] d t=1\right)$. The integration variable $t$ is component age, which runs from zero to infinite. Here $d g$ carries the mixed-layer signal $t$ years prior to $t_{\text {obs. }}$. Note that (1) is valid for any stable transient tracer.

It is advantageous to remove the dependence of $c_{m . l}$ on temperature and salinity by expressing CFC concentrations $c$ (in picomoles per kilogram) as an equivalent partial pressure $P$ (in parts per trillion by volume (pptv)). The relationship is $P=$ $c / F(T, S)$, with $F$ equal to the CFC solubility function [Wamer and Weiss, 1985]. $F$ appreciably decreases with temperature, whereas dependence on salinity can be regarded as secondary under oceanic conditions. As we assume solubility equilibrium for the mixed layer, (1) expressed in CFC partial pressure $P$ becomes

$$
P\left(t_{\mathrm{obs}}\right)=\int_{0}^{\infty} \frac{F[T(t), S(t)]}{F\left(T_{\mathrm{obs}}, S_{\mathrm{obs}}\right)} P_{\mathrm{atm}}\left(t_{\mathrm{obs}}-t\right) d g
$$

where $P_{\text {atm }}\left(t_{\text {obs }}-t\right)$ is the atmospheric partial pressure and $T(t), S(t)$ are the temperature and salinity of the component $d g(t)$. The age components $d g(t)$ now carry a weighting factor $F[T(t), S(t)] / F\left(T_{\text {obs }}, S_{\text {obs }}\right)$, which essentially gives added weight to colder components compared to warmer ones; the factor would be unity in a hypothetical isothermal and isohaline ocean. We note that under common oceanic conditions, the range of component temperatures will sufficiently restricted that $F$ can be taken to depend on temperature linearly; $T_{\text {obs }}$ and $S_{\text {obs }}$ are linear mixtures of the temperature and sa- linity of the parcel components. It follows that the average weighting factor will be approximately unity.

Because our intention is to address temporal evolution of oceanic CFC concentrations in quite general terms, we leave these subtleties (and also effects of a mixed layer-atmosphere solubility disequilibrium) to future consideration and restrict our treatment in the following to a simplified form of (2) in which the weighting factor is ignored; that is, we use

$$
P\left(t_{\mathrm{obs}}\right)=\int_{0}^{\infty} P_{\mathrm{atm}}\left(t_{\mathrm{obs}}-t\right) d g(t)
$$

Certain specific age distributions are introduced in the next section. The presentation uses an integrated form, i.e., $g(t)=$ $\int_{0}^{t}\left(d g / d t^{\prime}\right) d t^{\prime}$, which gives the fraction younger than $t$.

The atmospheric input function $P_{\text {atm }}(t)$ is well known since the late 1970 s because of the daily monitoring of these substances in the Atmospheric Lifetime Experiment Program (ALE) [Cunnold et al., 1986, 1994] since that time. Previous atmospheric contents have been evaluated using production rates and assumed emissions [Chemical Manufactures Association, 1983], with errors not exceeding approximately $\pm 10 \%$ [Warner and Weiss, 1992]. Yearly data from a listing of R. F. Weiss and P. K. Salameh (personal communication, 1992) for the southern hemisphere are presented in Figure 1; the same compilation shows that in recent years, northern hemisphere values are but a few percent higher. Atmospheric increases of both compounds apparently have been nearly exponential until 1974 and about linear since. Analytical approximations of the data, composed of exactly such functions, are shown in Figure 1 as solid lines. The partial pressures are well represented by the approximations, while there are deviations in the ratio of the two compounds, in particular before 1958. During the latter period, however, the partial pressures were small so that the deviation has only a small effect in practice.

We express the analytical functions by taking time relative to 1974; that is, we set $t_{0}=t_{\mathrm{obs}}-1974$. The functions are given in Table 1.

The analytical form of (3) becomes

$$
\begin{gathered}
P\left(t_{0}\right)=P_{0}\left\{\int_{0}^{t_{0}}\left[1+b\left(t_{0}-t\right)\right] d g(t)+\int_{t_{0}}^{\infty} e^{a\left(t_{0}-t\right)} d g(t)\right\} \\
t_{0}>0 \\
P\left(t_{0}\right)=P_{0}\left[\int_{0}^{\infty} e^{a\left(t_{t}-t\right)} d g(t)\right] \quad t_{0} \leq 0
\end{gathered}
$$

(Note that in the case of $t_{0}>0$ in the first integral the difference $\left(t_{0}-t\right) \geq 0$ and in the second integral $\left(t_{0}-t\right) \leq 0$.)

For illustration of implications of (4), consider two special cases. Suppose, first, that all components of a water parcel left the mixed layer prior to 1974 . In this case, all age components belonging to the term with the time-linear integrand in (4) vanish, so that, for a given water parcel (i.e., age distribution), $P\left(t_{0}\right)$ in our approximation is proportional to exp $\left(a t_{0}\right)$. This case of an exponential increase is expected to hold primarily for low CFC concentrations in deep advective regimes in the ocean. Prior to 1974 the atmospheric partial pressure likewise was proportional to $\exp \left(a t_{0}\right)$ (Table 1$)$. The apparent saturation of the parcel, defined as the observed partial pressure 


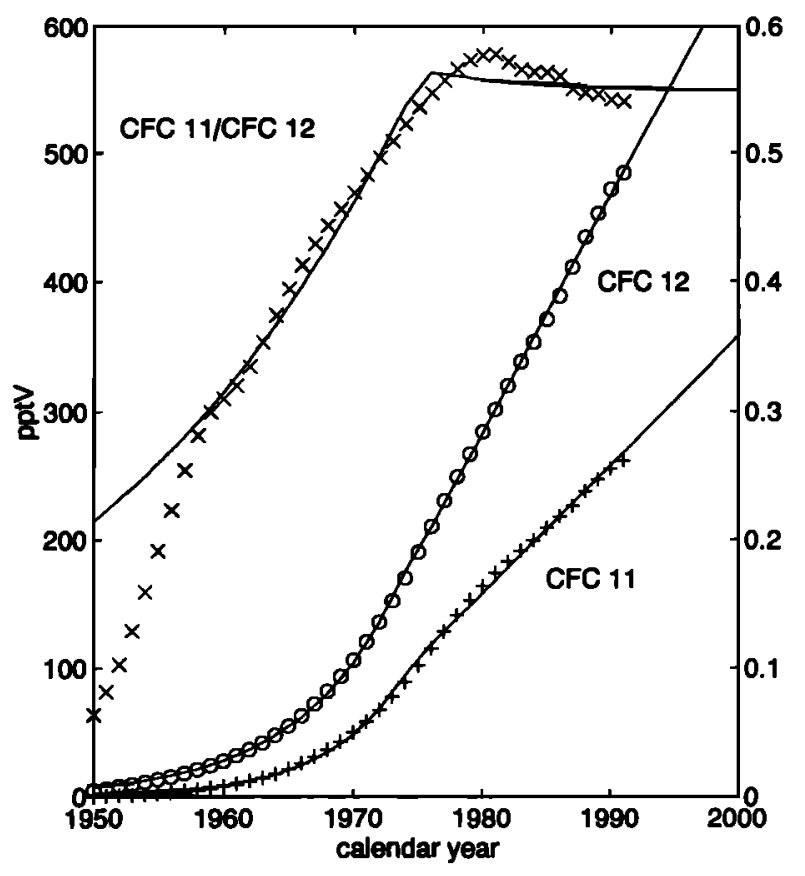

Figure 1. Tropospheric partial pressures (parts per trillion by volume) of CFC 11 (plusses) and CFC 12 (open circles) and CFC 11/CFC 12 partial pressure ratio (crosses), versus calendar year, starting in 1950, from a compilation by R. F. Weiss and P. K. Salameh (personal communication, 1992). The solid lines represent the analytical approximations to this data that we employ (see Table 1, but time is converted back to calendar year), extrapolated up to the year 2000. Data shown are for the southern hemisphere; northern hemisphere values are about $5 \%$ higher (more during the early period).

divided by the concurrent atmospheric partial pressure $\left[P\left(t_{0}\right) /\right.$ $P_{\text {atm }}\left(t_{0}\right)$ ], was thus constant in time up to 1974 .

The other case is that all components of a water parcel left the surface after 1974. Then the term in (4) with the exponential integrand vanishes, and concentration increases for both CFC 11 and CFC 12 are simply linear in time. The more general, mixed case with contributions from both before and after 1974 is addressed below.

Oceanic CFC measurements in actual fact started only after 1974 (i.e., $t_{0}>0$ ). In practice, therefore, the apparent saturation in our approximation is always given by

$$
\frac{P\left(t_{0}\right)}{P_{\mathrm{atm}}\left(t_{0}\right)}=\frac{\int_{0}^{t_{0}}\left[1+b\left(t_{0}-t\right)\right] d g(t)}{\left(1+b t_{0}\right)}+\frac{e^{a t_{0}} \int_{t_{0}}^{\infty} e^{-a t} d g(t)}{\left(1+b t_{0}\right)}
$$

The main aspect of this equation is that the apparent saturation increases monotonically in time: Any given age component $d g(t)$ will move up in calendar year as $t_{0}$ increases. The contribution of this component to the apparent saturation is proportional to $P_{\text {atm }}\left(t_{0}-t\right) / P_{\text {atm }}\left(t_{0}\right)$ (equation (3)). Because the fractional yearly increases in atmospheric partial pressure have been decreasing monotonically since 1974 (Figure 1), the value of this contribution increases with $t_{0}$, and the same must hold after integrating over the components $d g(t)$.

Eventually, components that are significantly different from zero will be found only in the first term on the right-hand side of (5). The contribution of a given age component to the apparent saturation will then be $\left\{\left[1+b\left(t_{0}-t\right)\right] /(1+\right.$ $\left.\left.b t_{0}\right)\right\} d g(t)$ (equation (5)), for which, as the age $t$ is fixed, the increase with $t_{0}$ is evident. In the long run, $t \ll t_{0}$ will hold for all components $d g(t)$ of a significant value. Consequently, the apparent saturation will gradually approach unity. In reality, (5) should underestimate the future rise in saturations, because atmospheric CFC 11 and CFC 12 concentrations are expected to level off, rather than continuing their present linear increase as (5) assumes [Cunnold et al., 1994].

\section{Selected Age Distributions and Resulting CFC Time Curves}

Age distributions of water parcels in the ocean can be of any form. As a next step we address four special ones that we consider to be characteristic of, or limiting cases for, coherent water bodies such as the central and intermediate waters of the ocean.

The age distributions chosen are (1) one of purely advective flow ("pipe flow"), i.e., uniform age of a water parcel; (2) age uniformly distributed between 0.5 and 1.5 of mean age (rectangular distribution); (3) age continuous between zero and several times the mean age, the actual form being chosen arbitrarily to meet our preconceptions of an age distribution typical of central waters; and (4) one of transport solely by mixing in one spatial coordinate (transport equation $\partial c / \partial t=$ $k\left(\partial^{2} c / \partial z^{2}\right)$, where $k$ is the eddy diffusivity and $z$ is the spatial coordinate, e.g., depth). Figure 2 presents the age distributions in integrated form (see section 2) normalized to a characteristic age, and Table 2 lists the functional forms. The charac-

Table 1. Form of Analytical Approximation Functions and Listing of Coefficients, for the Input Functions of CFC 11 and CFC 12

\begin{tabular}{llcc}
\hline & & $t_{0} \leq 0$ & $t_{0}>0$ \\
& & $P_{\text {atm }}=P_{0} \exp \left(a t_{0}\right)$ & $P_{\text {atm }}=P_{0}\left(1+b t_{0}\right)$ \\
\hline CFC 11 & $P_{0}=96.8986$ & $a=0.1694$ & $b=0.1032$ \\
CFC 12 & $P_{0}=174.6257$ & $a=0.1312$ & $b=0.1055$ \\
\hline
\end{tabular}

Time is in years; zero time corresponds to 1974. The $e$-folding scales for CFC 11 (CFC 12) before 1974 are 5.9 (7.6) years. As the comparison to observations below (section 4) uses southern hemisphere CFC data, the functions represent southern hemisphere values; increasing $P_{0}$ by $5 \%$ yields values about representative for the northern hemisphere. 


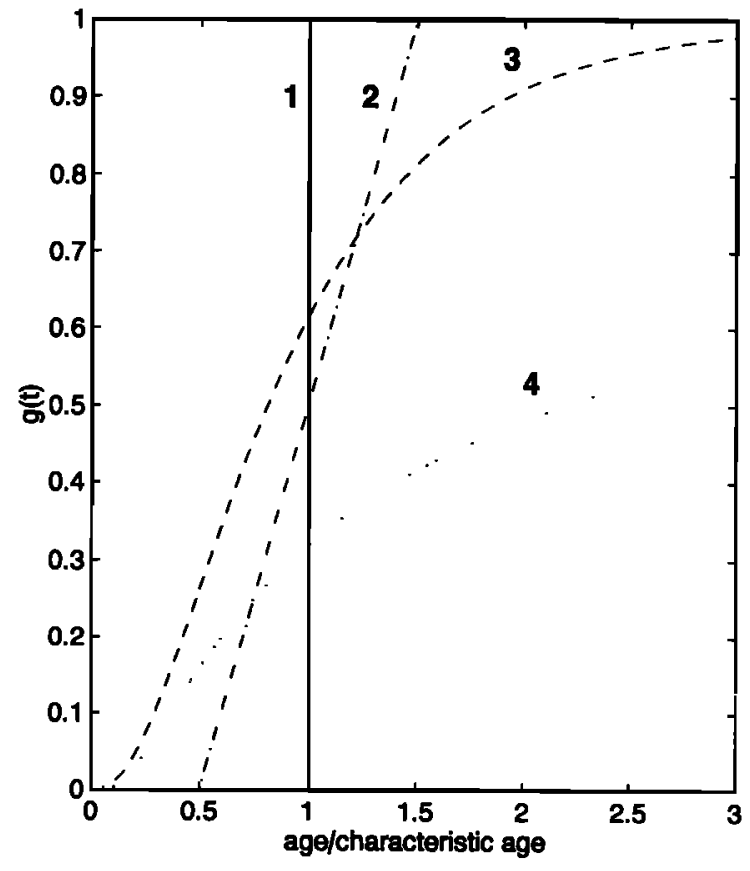

Figure 2. Time-integrated age distributions $g(t)$ (i.e., $g(t)=$ fraction of water in a parcel with age smaller than abscissa value, or $g(t)=\int_{0}^{t}\left(d g / d t^{\prime}\right) d t^{\prime} ; \int_{0}^{\infty}(d g / d t) d t \stackrel{=}{=}$ 1) for the four cases considered, versus age $t$ normalized by characteristic age (Table 2), see text. Case 1 (solid line) is the purely advective or piston-flow case; that is, all components have the same age. Case 2 (dashed-dotted line) has distribution constant between 0.5 and 1.5 times the characteristic age and vanishing elsewhere. Case 3 (dashed line) shows continuous age distribution. Case 4 (dotted line) is the onedimensional pure-mixing case.

teristic age for distributions $1-3$ is simply the mean age (Table 2). Distribution 4, which is derived in the Appendix, is more complex as a mean age cannot be defined. Instead, a displacement age (i.e., the time required to bring about a given mean square displacement from the mixed layer into the interior) is used, which agrees with the mean age in the case of purely advective flow.

Figures 3a-3i present CFC time curves resulting from the four distributions, for three different characteristic ages. The values chosen $(10,20$, and 50 years) supposedly span the timescales typical of central and intermediate waters, as well as of those portions of deep and bottom waters in the ocean in which current CFC concentrations are significantly above detection limits. Brief comments on the time curves follow.

Case 1: Pipe flow. As all constituents of a water parcel have uniform age, the concentrations as well as the CFC 11 to CFC 12 ratios simply follow the input functions with a time lag corresponding to the age. For long characteristic age the partial pressures, apparent saturations, and CFC 11 to CFC 12 ratios are lowest among those of the four distributions. This arises from the lack of any contributions younger than the mean age.

The apparent saturation starts with constant values and quickly rises after 1974 when the mixed-layer concentration increase changes from exponential to linear, up to a point when the increase of the interior concentration undergoes the same change (in Figure 3b 10 years after 1974, i.e., in 1984). Hereafter the rate of increase is reduced. At the same time the CFC 11 to CFC 12 ratio becomes nearly constant, after passing through a modest peak (note, however, that our approximation does not quite reproduce the temporal evolution of the actual CFC 11 to CFC 12 ratios (Figure 1)). Naturally, the peak is not present for any of the other distributions, because in these, components of a range of ages mix.

Case 2: Rectangular age distribution. For short characteristic age (Figures $3 a, 3 b$, and $3 c$ ) the time curves are quite similar to those of case 1, but as mentioned the peak in CFC 11 to CFC 12 ratio in 1984 is lost. For larger characteristic age the portion with ages less than the characteristic one causes values to be appreciable higher than for case 1 .

Case 3: Continuous age distribution. Throughout Figure 3 this case gives the highest values among the four distributions, except for the mentioned ratio maximum for case 1 (Figures $3 \mathrm{c}$ and 3f). This finding arises from the fact that distribution 3 has the highest contribution of ages below the characteristic one (Figure 2).

Case 4: One-dimensional mixing. For this distribution, concentration and saturation are found to be low for low characteristic age (Figures $3 a$ and $3 b$ ) relative to those of the other distributions but comparably higher for high characteristic age (Figures $3 \mathrm{~g}$ and $3 \mathrm{~h}$ ). This is due to a substantial contribution of ages younger than the characteristic one in the latter case and to a large one of much higher age in the former. Correspondingly, a crossover with the curves of the other age distributions is found for the intermediate characteristic age (Figures $3 \mathrm{~d}$ and 3e). CFC 11 to CFC 12 ratios are essentially identical to case 3 , independent of characteristic age. This feature arises because distribution 4 happens to be near to a composite of about $60 \%$ according to distribution 3 , complemented by much older

Table 2. Functional Form and Characteristic Age ( $\left.t_{\text {char }}\right)$ of Age Distributions

\begin{tabular}{lc}
\hline \multicolumn{1}{c}{ Age Distribution } & Characteristic Age \\
\hline $1 \quad d g / d t=\delta\left(t-t_{\text {age }}\right)$ & $t_{\text {char }}=\int_{0}^{\infty}(d g / d t) t d t=t_{\text {age }}$ \\
$2 d g / d t=1 / t_{\text {age }} \quad$ for $0.5 t_{\text {age }} \leq t \leq 1.5 t_{\text {age }} ;$ otherwise $d g / d t=0$ & $t_{\text {char }}=\int_{0}^{\infty}(d g / d t) t d t=t_{\text {age }}$ \\
$3 \frac{d g}{d t}=\frac{a t^{3}}{\exp (b(\sqrt{t}-1))}$ & $t_{\text {char }}=\int_{0}^{\infty}(d g / d t) t d t=71.76 / b^{2}$ \\
where $b$ is constant and $a=b^{8} / 10121$ & \\
$4 \frac{d g\left(t_{d}, t\right)}{d t}=\frac{1}{\sqrt{2 \pi}} \frac{t_{d}^{1 / 2}}{t^{3 / 2}} \exp \left(-t_{d} / 2 t\right)$, & $t_{\text {char }}=\left\langle z^{2}\right\rangle / 2 k=t_{d}$ \\
where $z$ is depth and $k$ is eddy diffusivity & \\
\hline
\end{tabular}

Time is in years; see text. 

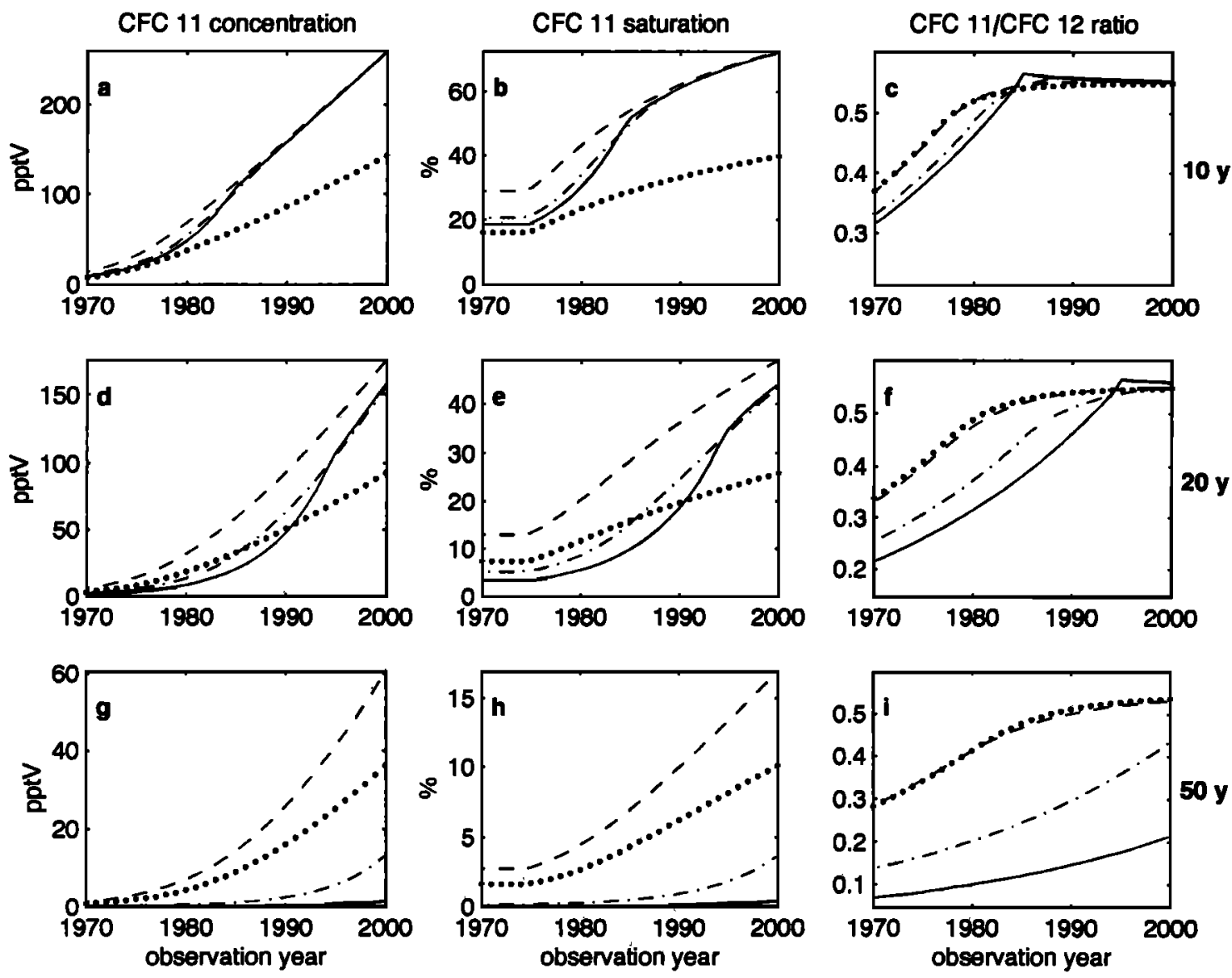

Figure 3. Theoretical CFC time curves for the period 1970-2000 according to (4) for the four age distributions considered (Table 2, Figure 2), with input functions from Table 1 and characteristic ages of 10, 20, and 50 years, respectively, see text. Case 1 is indicated by the solid lines, Case 2 by the dashed-dotted lines, Case 3 by the dashed lines, and Case 4 by the dotted lines. The first column (Figures 3a, 3d, and $3 \mathrm{~g}$ ) shows the CFC 11 partial pressure for characteristic ages of 10,20 , and 50 years. Note different scales. The second column shows (Figures $3 \mathrm{~b}, 3 \mathrm{e}$, and $3 \mathrm{~h}$ ) the same for apparent CFC 11 saturation. Note different scales. The third column (Figures 3c, 3f, and 3i) shows same for CFC 11/CFC 12 partial pressure ratio. Note differently expanded scales.

components which are essentially CFC free and will thus not alter the ratio.

A notable feature of distributions $1-3$ is that concentrations and saturations, for 10 years characteristic age, by about 1990 converge to a single curve (Figures $3 a$ and $3 \mathrm{~b}$ ). Approach to a similar situation beyond the year 2000 is indicated also for the intermediate characteristic age (Figures $3 d$ and $3 e$ ). Such convergency will necessarily occur once essentially all components of a water parcel are subjected to a linear increase of CFC concentration with time, so that the values depend solely on the characteristic age. Distribution 4 has a large portion of old components and therefore lags much behind the other distributions in this respect. The convergence formally follows from the fact that only the first term on the righthand side of (4) remains, which yields the concentrations as $P_{0}\left[1+b\left(t_{0}-t_{\text {char }}\right)\right]$, i.e., obviously independent of the age distribution.

One furthermore notes that the apparent saturations change distinctly in time for all four distributions. A typical rate of change in Figure 3 is $5-10 \%$ per decade. This means that for low saturations (Figure $3 \mathrm{~h}$ ), the relative changes are particularly large.
In the case of the CFC 11 to $\mathrm{CFC} 12$ ratios the convergence includes distribution 4, for reasons explained above. Moreover, an essentially constant value is being approached, independent of the characteristic age (Figures $3 \mathrm{c}$ and $3 \mathrm{f}$ ). The conclusion is that the ratios currently have ceased to provide much information on either a timescale or an age distribution, for characteristic ages of up to about 20 years. According to Figure 3i the situation appears to be more favorable for distinctly larger characteristic age. The useful range of ratios, however, is smaller than might appear from Figure 3i, because for ratios below about 0.4 , partial pressures are generally too low to allow measurement of the ratios at a useful precision. There is therefore even for characteristic ages sufficiently long, only a limited range of useful ratios, between large experimental uncertainties due to partial pressures being too low on the one hand and ratios becoming virtually constant on the other.

\section{Comparison to Observations}

Our issue is a prediction of the temporal evolution of the oceanic distributions of CFC 11 and CFC 12. A comparison to oceanic CFC observations is in order, to verify that our results, 


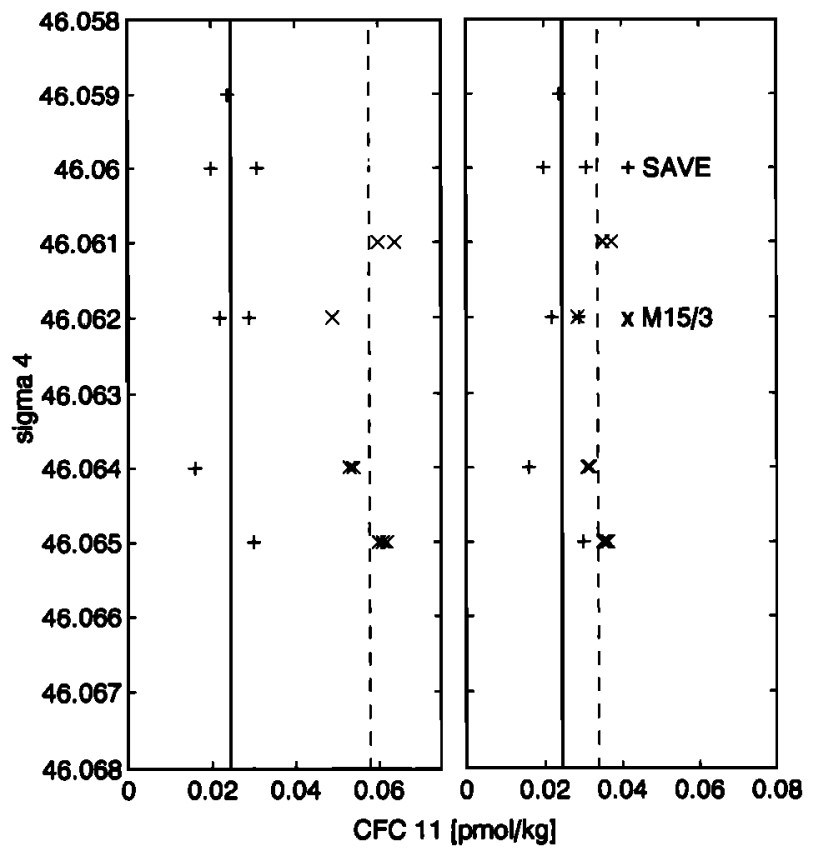

Figure 4a. Comparison of repeated CFC 11 observations in near-bottom waters. (left) CFC 11 concentration versus $\sigma_{4}$ in Antarctic Bottom Water in the Brasil Basin near $20^{\circ} \mathrm{S}$. South Atlantic Ventilation Experiment (SAVE) (1988) stations [Weiss et al., 1993] (plusses): $93\left(17.56^{\circ} \mathrm{S}, 28.69^{\circ} \mathrm{E}\right), 94\left(17.76^{\circ} \mathrm{S}\right.$, $\left.29.89^{\circ} \mathrm{E}\right)$, and $95\left(18.00^{\circ} \mathrm{S}, 30.98^{\circ} \mathrm{E}\right)$. Meteor $(1991)$ stations $\mathrm{M}$ $15 / 3$ (crosses): $142\left(19.00^{\circ} \mathrm{S}, 30.55^{\circ} \mathrm{E}\right), 143\left(19.00^{\circ} \mathrm{S}, 30.00^{\circ} \mathrm{E}\right)$, and $146\left(19.00^{\circ} \mathrm{S}, 28.44^{\circ} \mathrm{E}\right)$. (right) The same data, but $\mathrm{M} 15 / 3$ CFC 11 concentrations being time corrected to the year 1988 assuming exponential growth with a timescale as in Table 1.

such as those summarized in Figure 3, can provide useful guidance for the actual oceanic distributions. One aspect in this is to find out whether our assumption of a stationary ocean is sufficiently realistic. For the comparison we have selected a few cases from the limited number of repeated oceanic CFC observations that are available.

Figures $4 \mathrm{a}$ and $4 \mathrm{~b}$ give examples of observations in deep advective cores, sampled repeatedly in the course of up to 8 years. The cases considered are near-bottom waters in the Brasil Basin (Figure 4a) and in the Antarctic Circumpolar Current region in the western Agulhas Basin (Figure 4b). A note of caution is due inasmuch as the positions of the stations that we compare do not exactly agree, but as we plot the data in Figure 4 versus density (and in fact find little change with density), our station distances of about $100 \mathrm{~km}$ should be acceptable. The observed concentrations exhibit a distinct rise (Figures 4a and 4b, left diagrams), in keeping with an exponential increase postulated for such deep cores above (section 2; case of all components older than 1974). In fact, the observations within data errors collapse to single lines (Figures 4a and $4 \mathrm{~b}$, right-hand diagrams) if the later observations are corrected backward in time to the period of the first one, using an exponential time change with a timescale according to Table 1. The comparison thus supports our theoretical concepts and indicates that effects of nonstationarity are small. We note in passing that concentrations in particular in Figure 4a are quite low and were measured by different groups. The agreement therefore at the same time gives evidence that even such low data can be considered reliable.
Figure 5 presents data on the temporal increase of apparent saturations in the upper waters, the observations spanning about a decade. Shown are data through the central waters and into the Antarctic Intermediate Water (AAIW) at $30^{\circ} \mathrm{S}$ near to the Greenwich Meridian, as a function of potential density. According to the TS diagram shown in the inset, the stations selected are hydrographically rather indistinguishable. The main point in Figure 5 is that the apparent saturations do increase monotonically in time, quite as theoretically expected. In the central water range, moreover, the relative time change in saturation appears to be largest for lower saturations (near $\sigma_{0}=27.1$, there is roughly a doubling of saturation from 1983 to 1993), smaller further up, and generally of the order of $10 \%$ per decade, again as predicted. The temporal increase is less distinct in the AAIW range $\left(\sigma_{0}>27.15\right)$, and the temporal change appears to be somewhat uneven in time (the increase is relatively smaller between the 1989 and 1993 observations). We presume that here the limits posed by nonstationarity of ocean circulation are met.

A somewhat different approach is presented in Figure 6: The data shown are a scatter plot of CFC 11 to CFC 12 ratio versus CFC 11 partial pressure, observed in 1991 on a transect across the South Atlantic in $19^{\circ} \mathrm{S}$ (FS Meteor cruise M15/3) and essentially covering the central and intermediate waters. A small dynamic range of partial pressure ratios is evident in the data (a value of 0.45 appears to be a lower bound to ratios that can be determined with a useful precision). The average trend of ratios versus partial pressure is such that, above $100 \mathrm{pptv}$, the

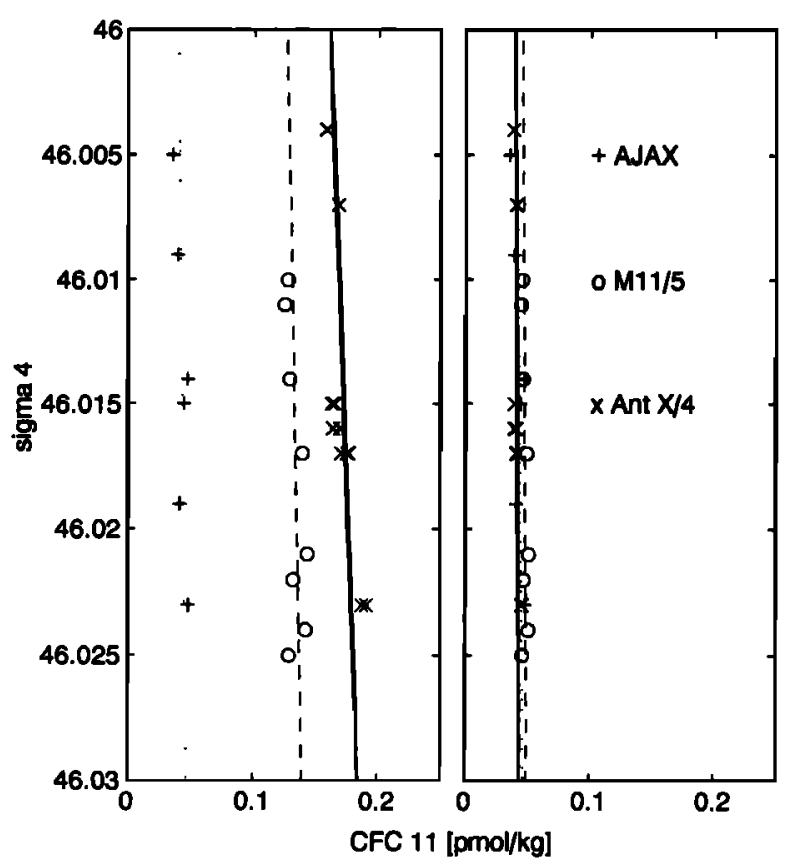

Figure 4b. (left) As in left panel of Figure 4a, but in bottom water between the Polar and Subantarctic Fronts near the Greenwich Meridian. Ajax (1984) stations [Weiss et al., 1990] (plusses): $69\left(47.00^{\circ} \mathrm{S}, 1.30^{\circ} \mathrm{E}\right), 71\left(48.00^{\circ} \mathrm{S}, 1.32^{\circ} \mathrm{E}\right)$, and 72 $\left(48.99^{\circ} \mathrm{S}, 1.17^{\circ} \mathrm{E}\right.$ ). Meteor (1990) stations cruise M 11/5 (open circles): $157\left(51.88^{\circ} \mathrm{S}, 3.35^{\circ} \mathrm{E}\right), 158\left(51.16^{\circ} \mathrm{S}, 3.79^{\circ} \mathrm{E}\right)$, and 160 (49.50 $\left.0^{\circ}, 4.75^{\circ} \mathrm{E}\right)$. Polarstern (1992) stations, cruise Ant X/4 (crosses): $568\left(46.77^{\circ} \mathrm{S}, 0.61^{\circ} \mathrm{E}\right), 570\left(48.00^{\circ} \mathrm{S}, 0.00\right), 571$ $\left(50.00^{\circ} \mathrm{S}, 0.04^{\circ} \mathrm{E}\right)$, and $572\left(50.50^{\circ} \mathrm{S}, 0.00\right)$. (right) Meteor and Polarstern data time corrected as in Figure 4a, right panel. 
ratio varies by no more than about $4 \%$. Such small variation for rather young waters was predicted above.

The crosses in Figure 6 are theoretical values obtained from age distribution 3 (Figure 2), with the characteristic age varying as noted. The solid line gives the input function (with the year of input indicated) and at the same time represents the piston-flow case (distribution 1). Piston flow obviously gives a poor fit to the data, the ratios being too high at intermediate partial pressures and too low below 80 pptv. The data for distribution 3 , on the other hand, reproduce the general shape of the scatter plot quite well, while only the ratios beyond about 5 years characteristic age are somewhat too high. Inspection of Figure 3 indicates that a distribution with young components being reduced somewhat relative to distribution 3 , which would produce lower ratios, should provide a good fit to the data.

The differences between the time marks at the piston flow line in Figure 6 and 1991, the year of the observations, correspond to piston-flow ages (e.g., 11 years for time mark 1980). One finds that piston-flow ages and characteristic ages from distribution 3 that correspond to a given CFC 11 concentration agree up to ages of nearly 15 years, while for larger ages they diverge appreciable.

\section{Discussion and Conclusions}

From the comparison to oceanic observations presented in the preceding section we conclude that our concept gives a

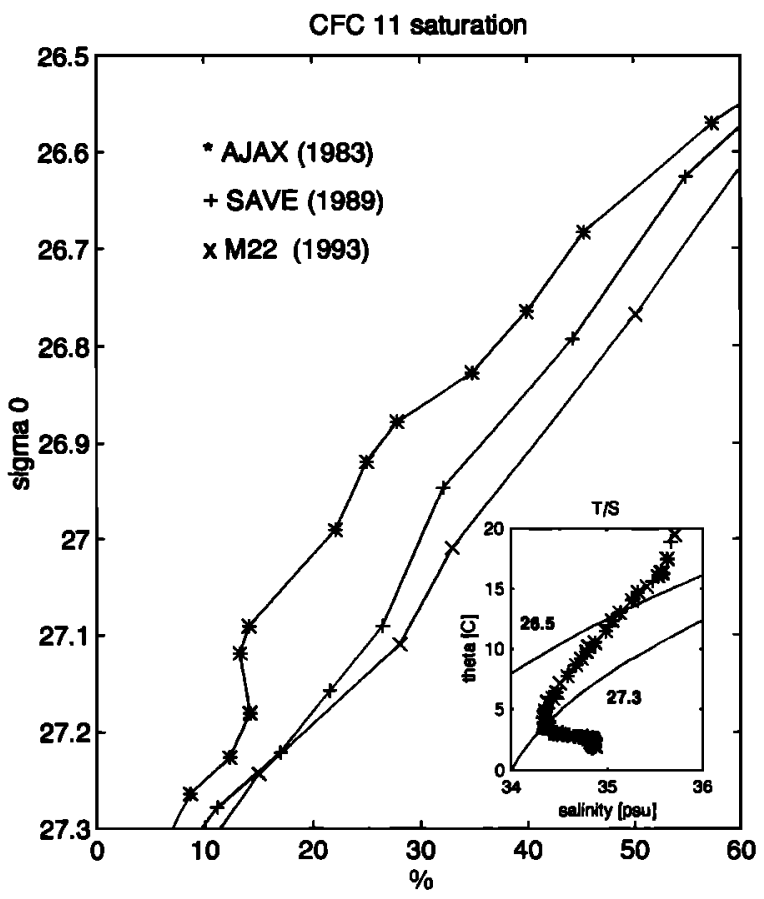

Figure 5. Temporal increase of apparent CFC 11 saturations over a range in potential density covering the central waters and reaching into Antarctic Intermediate Water, observed in the South Atlantic near $30^{\circ} \mathrm{S}$ just east of the Greenwich Meridian. Ajax (1983) station [Weiss et al., 1990] (asterisks) 38 $\left(1.83^{\circ} \mathrm{E}, 30.00^{\circ} \mathrm{S}\right), \mathrm{SAVE}(1989)$ station [Weiss et al., 1993] (plusses) $220\left(2.01^{\circ} \mathrm{E}, 29.99^{\circ} \mathrm{S}\right)$, and Meteor M22/5 (1993) station (crosses) $74\left(0.57^{\circ} \mathrm{E}, 29.86^{\circ} \mathrm{S}\right)$ are shown. The positions for the first two stations agree well, but the Meteor station is about $150 \mathrm{~km}$ to the west. Inset shows combined TS diagram of the three stations with isopycnals $\sigma_{0}=26.5$ and $\sigma_{0}=27.3$ shown.

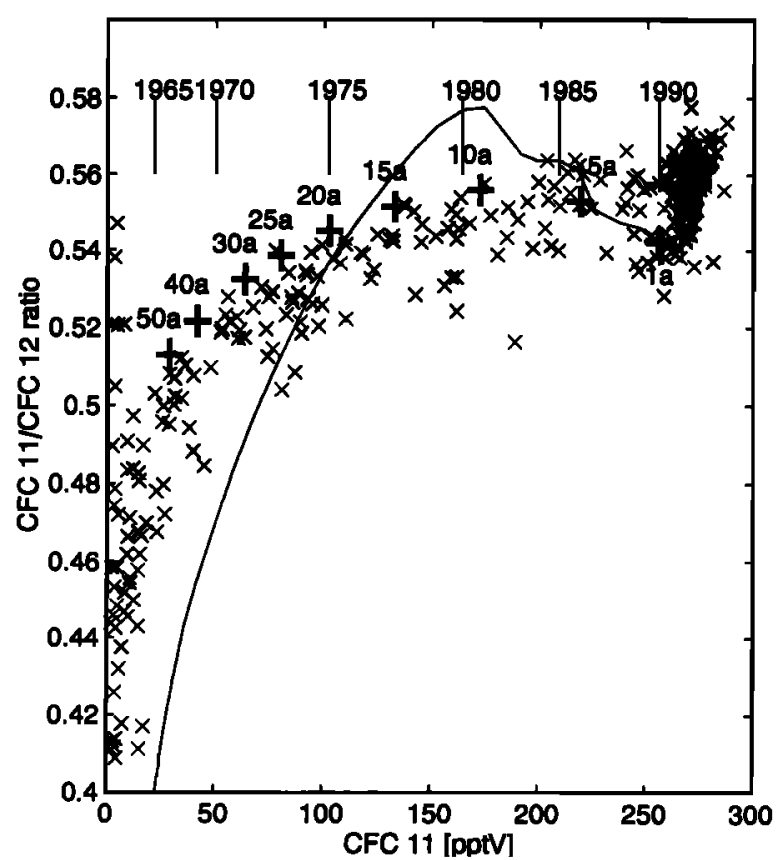

Figure 6. Scatter plot of CFC 11/CFC 12 partial pressure ratios versus CFC 11 partial pressures (crosses), measured along a zonal transsect in $19^{\circ} \mathrm{S}$ in the South Atlantic (Meteor cruise M15/3, 1991). The solid line represents the atmospheric input function; the appropriate years are indicated in the upper part of the figure; as the observations were made in 1991, a 10 -year age corresponds to 1981 . The plusses give values according to age distribution 3 for characteristic ages as indicated (see Figure 2, Table 2, and text). For better comparability with the observations the theoretical values are based on the true input data rather than on the analytical approximations (Figure 1).

useful framework to assess the temporal evolution of CFC 11 and CFC 12 concentrations in the ocean. Figure 3 can provide quantitative guidance for the two applications mentioned in the introduction, namely, dealing with nonsynoptic data and constructing time-dependent CFC boundary conditions for inner-ocean boundaries of ocean circulation models. One should keep in mind that in the former case, the time intervals over which corrections for temporal change are required are usually restricted, so that the corrections are not too critical. Similarly, if inner-ocean CFC time curves over the entire CFC transient are to be constructed, the early concentrations are small enough that for them very accurate reconstructions are generally not required.

It is preferable to apply extrapolations in time, as required by these applications, to apparent saturations rather than concentrations, because time changes for the former are less. Still, apparent CFC saturations increase monotonically in time in the ocean, and for lower CFC concentrations the relative temporal increases are rather larger than for higher ones (Figure 3 ). These aspects are addressed in Figure 7, which gives time curves of apparent saturation of CFC 11 similar to Figures $3 b$, $3 \mathrm{e}$, and $3 \mathrm{~h}$, but with the characteristic ages being chosen such that saturations in 1994 attain prescribed values, i.e., 5, 20, and $60 \%$. Time curves normalized in this fashion give evidence on how much the temporal increases of apparent saturation depend on the actual age distribution. The piston-flow case (age distribution 1, Figure 2 ) is not believed to be of much relevance 

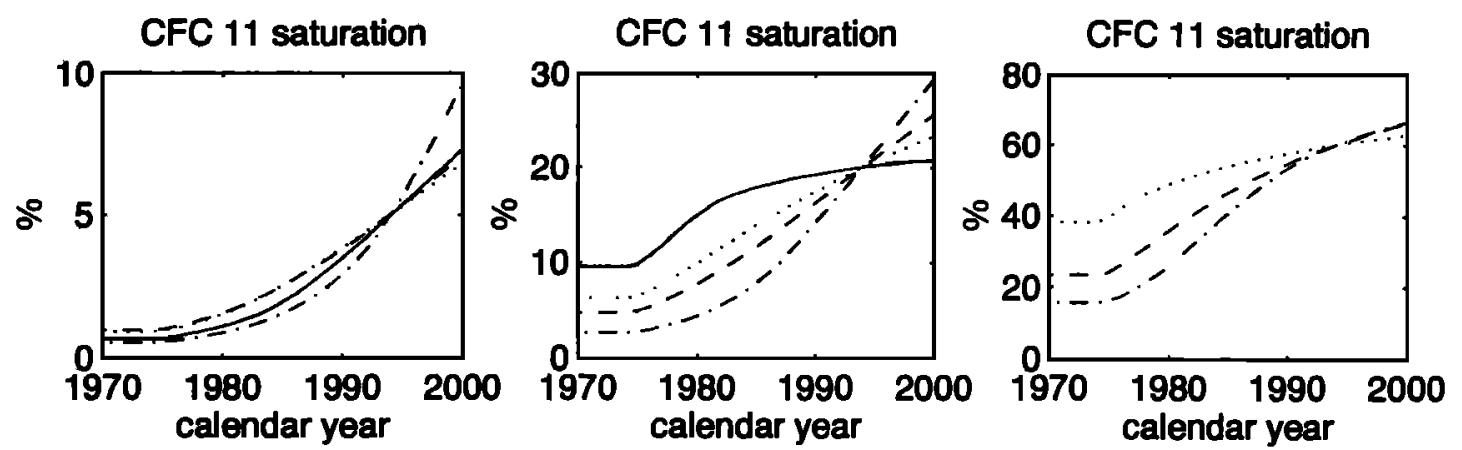

Figure 7. Time curves of apparent saturation for the period 1970-2000 according to (5) for different age distributions, with characteristic ages chosen such that saturations in 1994 attain prescribed values. Dasheddotted indicates distribution 2 , dashed line indicates distribution 3 , dotted line indicates distribution 4 , and solid line indicates distribution 2 but diluted by $75 \%$ CFC-free water. Note that meaning of solid line is different from Figure 3. (left) The 1994 apparent saturation 5\%; characteristic ages: distribution 2, 39.5 years; distribution 3, 82 years; distribution 4, 65 years; distribution 2, diluted, 25.5 years. (middle) The 1994 apparent saturation 20\%; characteristic ages: distribution 2, 25.4 years; distribution 3, 37.3 years; distribution 4, 22.7 years; distribution 2, diluted, 5.9 years. (right) The 1994 apparent saturation 60\%; characteristic ages: distribution 2, 11.9 years; distribution 3, 12.3 years; distribution 4, 2.7 years; distribution 2, diluted, cannot reach this saturation and is thus not shown.

in practice and is therefore not shown. A more useful case included instead in Figure 7 is a distribution consisting of $25 \%$ of distribution 2 diluted by $75 \%$ of CFC-free water.

In Figure 7, apparent saturations change from 1974, when in our approximation the values begin to increase, to 1994 by large factors. In all three diagrams, changes are largest for distribution 2, amounting to between about $25 \%$ per decade (right diagram, high 1994 saturation) and $2.5 \%$ per decade (left diagram, low 1994 saturation). They are smallest for distribution 4 in the right diagram and for distribution 2, diluted, in the center diagram. These are the distributions that have large portions of comparably old water (Figure 2), so that, in order to bring about the prescribed 1994 saturations, the turnover time of the young component ("characteristic age," see caption) becomes rather short. For the higher 1994 saturations (right and middle diagrams) the spread of saturations between the various age distributions is substantial (up to a factor of 4 in saturation in about 1980). A smaller spread is apparent for the low 1994 saturation (left diagram). This holds because the low saturations require turnover times so long that the coherent exponential increase in the source function prior to 1974 is still influential (see section 2). In this case, furthermore, saturations for age distribution 2, diluted, are lower than those for distributions 3 and 4, because the latter distributions include really young water (i.e., less than half the characteristic age, Figure 2), which the former lacks.

Figure 7 thus shows that temporal changes in apparent saturations are substantial and strongly depend on the age distribution of the water. For low saturations (Figure 7, left), relative changes in saturation are largest, but at the same time the influence of different age distributions appears to be less. The case of distribution 2, diluted, demonstrates that in the range of small apparent saturations, comparably smaller temporal increases can (Figure 7, center) be found if the low saturations are caused by dilution with CFC-free water, rather than by a long advective timescale (W. Roether et al., Spreading of newly formed deep waters along the northern rim of the Weddell Basin, submitted to Journal of Geophysical Research, 1994). In total, it is evident from Figure 7 that the concept of apparent saturations being time invariant [Gordon et al., 1992] can underestimate the time changes by up to about $20 \%$ per decade.

It is realized that age distributions in special situations may be quite different from the ones considered in this communication. Examples are mixtures of two or more deep water masses that carry significant CFC concentrations. Age distributions with more than one age peak might result. We believe that the present work can provide guidance also in such situations, for example, with the statement that an exponential concentration increase must prevail if all water masses involved are sufficiently "old" (section 2 ).

For the two applications mentioned the diagrams of Figure 7 can provide assistance in the following way: One guesses which one of the age distributions would be most appropriate in a given oceanographic situation and reads the time changes of the apparent saturation from the respective plot in Figure 7. As the three diagrams in Figure 7 give curves only for certain values of the current saturation, some interpolation between the three diagrams will generally be required. An alternative procedure would be to use a, possibly very basic, model of the water body in question [Thiele et al., 1986] to produce an approximate age distribution, for which a time curve corresponding to Figure 7, but directly related to the application in question, could be computed from (5). Even though time curves obtained either way will naturally have limited accuracy, if reasonable choices were made, they should represent a significant improvement over the mentioned concept of a timeinvariant apparent saturation used previously [Gordon et al., 1992].

Figure 6 above was an example that observations can point toward a certain age distribution of a water body. The age distribution is a product of the actual circulation and mixing fields and should thus give clues on them. Suitable ways to exploit this relationship should be explored. These might include a comparison of CFC data simulated by means of an ocean circulation model with observations in a plot like in Figure 6. It was furthermore noted above from Figure 6 that CFC concentrations based on the widely differing age distributions of piston flow and age distribution 3 (Figure 2) are 
nearly identical up to about 15 years. Such agreement is certainly a result of the quasi-linear concentration increase since about 1974 (Figure 1), and it adds credibility to ages based on CFC 11 (or CFC 12) concentrations. We repeat that such age has to be understood as the time since leaving the mixed layer, averaged over all components of a water parcel.

We have treated only the transient tracers CFC 11 and CFC 12. The reasons are that these are the CFCs for which by far the most data exist, also over an extended period in time, and that on the other hand the near similarity of their input functions, which might lead to a certain redundancy between the two CFCs, deserves scrutiny. We found that CFC 11 to CFC 12 ratios vary systematically in water bodies of a sufficiently long turnover timescale. The dynamical range of the ratios is restricted, however, because for very long timescales the ratios cannot be determined with sufficient precision. For shorter turnover timescales (about a decade or less), approach to an approximately constant ratio was noted (Figure 3). But also in this case, CFC 11 to CFC 12 ratios can provide useful diagnostics, for example, on the presence or absence of older components in a water parcel. In all such applications, high measurement precision is a prerequisite.

The concept of age distributions might be applied to other transient tracers as well, not the least to the CFCs CFC 113 and $\mathrm{CCl}_{4}$, for which oceanic observations have started to become available [Wallace et al., 1994]. As the input functions of these compounds are distinctly different from those of CFC 11 and CFC 12, data from all four CFCs, and possibly further transient tracers such as tritium and bomb ${ }^{14} \mathrm{C}$, would provide independent constraints on age distributions. Then it might be possible to lift the assumption of stationarity on which the present analysis is based and address also the variability of ocean circulation.

\section{Appendix: Age Distribution in the Case of Pure Mixing}

To obtain an age distribution for the case of pure mixing, consider a situation in which surface water is transferred into the interior ocean by mixing in one dimension, say $z$, only (Fickian diffusion, eddy diffusivity $k$ independent of $z$ ). We ask for the contribution of water that was equilibrated in the mixed layer with the atmosphere at a certain time $t^{*}$, attaining a tracer concentration $c\left(0, t^{*}\right)$, to the concentration $c_{\mathrm{obs}}\left(z, t_{\mathrm{obs}}\right)$ observed at a certain distance $z$ from the outcrop at a later time $t_{\text {obs. }}$. The answer is obtained through the solution for diffusion into a semiinfinite medium with time-dependent concentration at the boundary [Luikov, 1968]. For a mixedlayer tracer concentration in form of a delta function in time $\delta\left(t-t^{*}\right) c\left(0, t^{*}\right)$, one obtains the resulting fractional tracer distribution in the interior as

$$
\delta c^{*}\left(z, t_{\mathrm{obs}}\right)=\frac{z}{\sqrt{4 k \pi}} \frac{c\left(0, t^{*}\right)}{\left(t_{\mathrm{obs}}-t^{*}\right)^{3 / 2}} \exp \left[-z^{2} / 4 k\left(t_{\mathrm{obs}}-t^{*}\right)\right]
$$

Integrating the contributions for all $t^{*}$, which runs from infinitely old to $t_{\text {obs }}$, one obtains

$$
c\left(z, t_{\mathrm{obs}}\right)=\frac{z}{\sqrt{4 k \pi}} \int_{0}^{\infty} \frac{c\left(0, t_{\mathrm{obs}}-t\right)}{t^{3 / 2}} \exp \left(-z^{2} / 4 k t\right) d t
$$

where $t=t_{\mathrm{obs}}-t^{*}$; that is, $t$ represents the age of the water.
For a fixed distance $z$ from the outcrop, comparison with (A1) then yields for the age distribution

$$
[d g(z, t) / d t]=\left(z / \sqrt{4 k \pi t^{3 / 2}}\right) \exp \left(-z^{2} / 4 k t\right)
$$

CFC concentration profiles with distance from the outcrop obtained by entering this age distribution into (1) are identical with ones found previously by numerical solution of the transport equation for pure mixing [Roether et al., 1993].

Apparently, the semiinfinite medium provides water so old that (1) does not yield a finite mean age. To obtain a characteristic timescale also in this case, we take the analogy that in pure advection, an age $t_{d}$ is clearly related to a corresponding depth, namely, the mean displacement from the outcrop during $t_{d}$. The relationship between time and mean displacement from the outcrop in the pure-diffusion case is $\langle z\rangle=\sqrt{2 k t_{d}}$. Entering this condition, one has

$$
\frac{d g\left(t_{d}, t\right)}{d t}=\frac{1}{\sqrt{2 \pi}} \frac{t_{d}^{1 / 2}}{t^{3 / 2}} \exp \left(-t_{d} / 2 t\right)
$$

Acknowledgments. This work is part of the Ph.D. thesis of the first author, prepared at the Institut für Umweltphysik, Bremen, Germany. We are grateful to R. F. Weiss, SIO, La Jolla, for letting us use CFC data from the SAVE program and to A. Putzka and $K$. Bulsiewicz, University of Bremen, for their contributions to the CFC measurements on Meteor and Polarsterm. The measurements of the Bremen group were supported by the Bundesminister für Forschung und Technologie and the Deutsche Forschungsgemeinschaft, both in Bonn, Germany.

\section{References}

Bullister, J. L., Chlorofluorocarbons as time-dependent tracers in the ocean, Oceanography, 2, 12-17, 1989.

Bullister, J. L., and R. F. Weiss, Determination of $\mathrm{CCl}_{3} \mathbf{F}$ and $\mathrm{CCl}_{2} \mathrm{~F}_{2}$ in seawater and air, Deep Sea Res. Part A, 35, 839-853, 1988.

Chemical Manufactures Association, World Production and release of fluorocarbons 11 and 12 through 1982, report, Fluorocarbon Program Panel, Washington, D. C., 1983.

Cunnold, D. M., R. G. Prinn, R. A. Rasmussen, P. G. Simmonds, F. N. Alyea, C. A. Cardelino, A. J. Crawford, P. J. Fraser, and R. D. Rosen, Atmospheric lifetime and annual release estimates for $\mathrm{CFCl}_{3}$ and $\mathrm{CF}_{2} \mathrm{Cl}_{2}$ from 5 years of ALE data, J. Geophys. Res., 91(D10), $10,797-10,817,1986$.

Cunnold, D. M., P. J. Fraser, R. F. Weiss, R. G. Prinn, P. G. Simmonds, B. R. Miller, F. N. Alyea, and A. J. Crawford, Global trends and annual releases of $\mathrm{CCl}_{3} \mathrm{~F}$ and $\mathrm{CCl}_{2} \mathrm{~F}_{2}$ estimated from ALE/ GAGE and other measurements from July 1978 to June 1991, J. Geophys. Res., 99(D1), 1107-1126, 1994.

Doney, S. C., and J. L. Bullister, A chlorofluorocarbon section in the eastern North Atlantic, Deep Sea Res. Part A, 39, 1857-1883, 1992.

Gordon, A. L., R. F. Weiss, W. M. Smethie, and M. J. Warner, Thermocline and intermediate water communication between South Atlantic and Indian Oceans, J. Geophys. Res., 97(C5), 7223-7240, 1992.

Luikov, A. V., Analytical Heat Diffusion Theory, Academic, San Diego, Calif., 1968.

Pickart, R. S., N. G. Hogg, and W. M. Smethie Jr., Determining the strength of the Deep Western Boundary Current using the chlorofluoromethane ratio, J. Phys. Oceanogr., 19, 940-951, 1989.

Roether, W., R. Schlitzer, A. Putzka, P. Beining, K. Bulsiewicz, G. Rohardt, and F. Delahoyde, A chlorofluoromethane and hydrographic section across Drake Passage: Deep water ventilation and meridional property transport, J. Geophys. Res., 98(C8), 14,42314,435, 1993.

Roether, W., V. M. Roussenov, and R. Well, A tracer study of the thermohaline circulation of the Eastern Mediterranean, in Ocean Processes in Climate Dynamics: Global and Mediterranean Examples, edited by P. Malanotte-Rizzoli and A. R. Robinson, pp. 371-394, Kluwer Acad., Norwell, Mass., 1994. 
Smethie, W. M., Jr., Tracing the thermohaline circulation in the western North Atlantic using chlorofluorocarbons, Prog. Oceanogr., 31, 51-99, 1993.

Thiele, G., W. Roether, P. Schlosser, R. Kuntz, G. Siedler, and L. Stramma, Baroclinic flow and transient tracer fields in the Canary-Cape Verde Basin, J. Phys. Oceanogr., 16, 814-826, 1986.

Wallace, D. W. R., P. Beining, and A. Putzka, Carbon tetrachloride and chlorofluorocarbons in the South Atlantic $19^{\circ} \mathrm{S}, \mathrm{J}$. Geophys. Res., 99(C4), 7803-7819, 1994.

Warner, M. J., and R. F. Weiss, Solubilities of chlorofluorocarbons in water and seawater, Deep Sea Res. Part A, 32, 1485-1497, 1985.

Warner, M. J., and R. F. Weiss, Chlorofluoromethanes in South Atlantic Antarctic Intermediate Water, Deep Sea Res., 39, 2053-2075, 1992.

Weiss, R. F., J. L. Bullister, R. H. Gammon, and M. J. Warner, Atmospheric chlorofluorocarbons in deep equatorial Atlantic, $\mathrm{Na}$ ture, 314, 608-610, 1985.

Weiss, R. F., J. L. Bullister, M. J. Warner, F. A. van Woy, and P. K.
Salameh, AJAX Expedition chlorofluorocarbon measurements, S.I.O. Ref. 90-6, 109 pp., Scripps Inst. of Oceanogr., La Jolla, Calif., 1990.

Weiss, R. F., M. J. Warner, P. K. Salameh, F. A. van Woy, and K. G. Harrison, South Atlantic Ventilation Experiment: SIO chlorofluorocarbon measurements, SIO Ref. 93-49, 466 pp., Scripps Inst. of Oceanogr., La Jolla, Calif., 1993.

P. Beining, Institut für Meereskunde an der Universität Kiel, Dustembrooker Weg 20, 24105 Kiel, Germany. (e-mail: pbeining@ifm.unikiel.d400.de)

W. Roether, Institut für Umweltphysik der Universität Bremen, 28334 Bremen, Germany.

(Received November 1, 1994; revised September 25, 1995; accepted February 1, 1996.) 